40th "Jaszowiec" International School and Conference on the Physics of Semiconductors, Krynica-Zdrój 2011

\title{
Wide Range Wavelength Tuning of InGaAsP/InP Laser Diodes
}

\author{
M. BAJdA ${ }^{a, b, *}$, W. TRZeCiAKOWski ${ }^{b}$ AND J.A. MAJEWSKI ${ }^{a}$ \\ ${ }^{a}$ Institute of Theoretical Physics, Faculty of Physics, University of Warsaw \\ Hoża 69, 00-681 Warszawa, Poland \\ ${ }^{b}$ Institute of High Pressure Physics Unipress, Polish Academy of Sciences \\ Sokołowska 29/37, 01-142 Warszawa, Poland
}

\begin{abstract}
We present results of theoretical studies of external tuning for laser diodes based on InGaAsP/InP heterostructures at temperatures from $300 \mathrm{~K}$ down to $80 \mathrm{~K}$ and at hydrostatic pressures up to $2.27 \mathrm{GPa}$. The tuning range achieved by pressure and grating was $390 \mathrm{~nm}$ (from $1220 \mathrm{~nm}$ to $1610 \mathrm{~nm}$ ). At lower temperatures the tuning range achieved with grating was significantly reduced. Our results indicate that pressure tuning is much more effective than temperature tuning when combined with tuning by external grating.
\end{abstract}

PACS: $42.55 . \mathrm{Px}, 85.60 . \mathrm{Jb}$

\section{Introduction}

The most common method for wavelength tuning of the laser diode involves a wavelength-selective reflection of light in an external cavity system [1]. Such tuning is limited to the range of positive net gain of the laser. Another method consists in shifting the whole gain spectrum of the laser by the application of high pressure or low temperature [2] (increased temperatures usually increase the threshold currents and decrease the efficiency). High pressure and low temperature increase the direct bandgaps in the active layer of the laser, shifting its emission to shorter wavelengths.

In order to model the experimental results, we have performed calculations of gain as a function of pressure and temperature, employing an $8 \times 8 k \cdot p$ scheme [3] for electronic structure of the InGaAsP quantum well. The gain is obtained as a function of carrier concentration and the concentration, in turn, is related to current assuming two recombination channels: radiative and the Auger ones. Since the radiative recombination dominates at temperatures up to $220 \mathrm{~K}$, knowledge of the temperature dependence of the threshold currents allows us for separation of these two contributions.

\section{Theoretical model}

The fundamental characteristics of the quantum-well laser influencing its wavelength-tuning capability is the optical gain. It is determined by the electronic band structure (similarly to absorption) and by the carrier concentration in the quantum well (determined by pumping).

\footnotetext{
* corresponding author; e-mail: maziarz@fuw.edu.pl
}

The subband-to-subband optical gain can be calculated using the Fermi golden rule

$$
\begin{gathered}
g_{n m}(\hbar \omega)=\frac{\pi e^{2} \hbar}{n_{1} \varepsilon_{0} c m_{0}^{2} V} \frac{1}{\hbar \omega} \sum_{\boldsymbol{k}, \sigma}\left|\hat{\varepsilon} P_{n m}^{\sigma}(\boldsymbol{k})\right|^{2} \delta\left(E_{n}^{\mathrm{e}}(\boldsymbol{k})\right. \\
\left.-E_{m}^{\mathrm{h}}(\boldsymbol{k})-\hbar \omega\right)\left[f^{\mathrm{e}}\left(E_{n}^{\mathrm{e}}(\boldsymbol{k})\right)-f^{\mathrm{h}}\left(E_{m}^{\mathrm{h}}(\boldsymbol{k})\right)\right],
\end{gathered}
$$

where $V$ is the crystal volume, $\hat{\varepsilon}$ is the polarization of light, $\hbar \omega$ is the photon energy, $f^{\mathrm{e}}$ and $f^{\mathrm{h}}$ are the distribution functions for electron and holes, respectively, and $P_{n m}^{\sigma}(\boldsymbol{k})$ is the optical matrix element. To get the final material gain, we must integrate over the energy applying a broadening function

$$
g_{n m}(E)=\int \mathrm{d} E^{\prime} g_{n m}\left(E^{\prime}\right) L\left(E-E^{\prime}\right) .
$$

For broadening, we have used Lorentzian distribution

$$
L\left(E-E^{\prime}\right)=\frac{1}{\pi} \frac{\hbar / \tau_{\text {in }}}{\left(E-E^{\prime}\right)^{2}+\left(\hbar / \tau_{\text {in }}\right)^{2}},
$$

with intraband relaxation time $\tau_{\text {in }} \approx 0.1 \mathrm{ps}[4]$.

In narrow band gap materials good description of the band structure requires an $8 \times 8 k \cdot p$ model which includes the conduction band, the heavy and light-hole valence bands and the spin-orbit split-off band. For the quantum well, the model must also take into account the built-in strain (in case of lattice mismatch between the well and the barrier) and the proper boundary conditions for the envelope functions. We have used the nextnano ${ }^{3}$ software package [3] which yields the envelopes and dispersion within the nonparabolic $8 \times 8 \mathrm{k} \cdot \mathrm{p}$ model and then allows for calculation of the absorption profile of the quantum well. For a given carrier concentration in the quantum well (assuming charge neutrality i.e. $n=p$ ), we have calculated the quasi Fermi levels for electrons and holes. This, in turn, allows us to obtain the Fermi-Dirac occupation probabilities $f_{\mathrm{e}}$ 
and $f_{\mathrm{h}}$. The material gain has been obtained by multiplying the absorption profile by $f_{\mathrm{e}}-f_{\mathrm{h}}$. We neglect many-body effects and we assume flat-band conditions in the well and barrier. The information on the parameters of considered laser has been taken from the literature data on similar bent-waveguide lasers $[5,6]$. The active layer contains two $10 \mathrm{~nm} \operatorname{In}_{0.73} \mathrm{Ga}_{0.27} \mathrm{As}_{0.82} \mathrm{P}_{0.18}$ compressively strained $(0.9 \%)$ quantum wells surrounded by $\mathrm{In}_{0.73} \mathrm{Ga}_{0.27} \mathrm{As}_{0.57} \mathrm{P}_{0.43}$ lattice matched barriers and InP claddings. The width of the ridge is $5 \mu \mathrm{m}$, chips are $1 \mathrm{~mm}$ long.

TABLE

Material parameters at room temperature and zero pressure used in the calculations. In the first row the expression for the energy gap as a function of pressure and temperature is given.

\begin{tabular}{l|c}
\hline \hline$E_{\mathrm{g}}(p, T)$ & $0.8111+0.01094 p-0.000127 p^{2}-\frac{0.00034 T^{2}}{T+50}$ \\
$a-$ absolute deformation potential at $\Gamma$ point & $-5.1 \mathrm{eV}$ \\
$b, d-$ uniaxial deformation potentials for the valence band & $-1.769 \mathrm{eV},-3.753 \mathrm{eV}$ \\
$\gamma_{1}, \gamma_{2}, \gamma_{3}-$ Luttinger parameters & $17.56,7.274,7.984$ \\
$\varepsilon_{r}-$ dielectric constant in the active layer & 14.33 \\
$\Delta E_{\mathrm{c}}-$ conduction-band offset & $0.210 \mathrm{eV}$ \\
$\Delta E_{\mathrm{v}}-$ valence-band offset & $0.133 \mathrm{eV}$ \\
$n_{1}-$ refractive index in the active layer & 3.786 \\
$\Delta_{\text {so }}$ - spin-orbit splitting in the quantum-well material & $0.35 \mathrm{eV}$ \\
$E_{p}-$ energy equivalent of the dipole matrix element & $20.53 \mathrm{eV}$
\end{tabular}

Material parameters (bandgap, the Luttinger parameters, band-offsets, deformation potentials) have been taken from [5] and are listed in Table. The pressure and temperature dependence has been introduced through the variation of the direct energy gaps (in the well and in the barrier) with pressure and temperature

$$
E_{\mathrm{g}}(p, T)=E_{\mathrm{g}}^{0}+\alpha p+\beta p^{2}-\frac{\gamma T^{2}}{T+\delta},
$$

where we assume quadratic dependence of the bandgap on pressure and the Varshni dependence on temperature (see Table). We assume also that the pressure variation does not depend on temperature and vice versa.

The effective masses of electrons, light holes and spinorbit split-off holes change with pressure and temperature according to the $8 \times 8 k \cdot p$ model. The mass of heavy holes, the spin-orbit splitting and the band offsets are assumed independent of pressure and temperature. Apart from the dominant e1-hh1 transition we must include (in the calculation of gain) the e2-hh2 transition and the e1-lh1 transition (which are almost degenerate). Due to the compressive built-in strain, the light-hole levels are pushed away from the heavy hole levels. For low carrier concentrations, gain is mostly dominated by the hh1-e1 transition but we include in our calculations all three above-mentioned transitions.

Within the described model, we are able to obtain the material gain for a given carrier concentration in the well. The region of positive gain is determined by the Bernard-
Duraffourg condition $E_{\mathrm{g}}<\hbar \omega<E_{\mathrm{g}}+\Delta E_{\mathrm{f}}$, where $E_{\mathrm{g}}$ is the effective bandgap in the quantum well and $\Delta E_{\mathrm{f}}$ is the sum of the Fermi energies in the valence and conduction bands. In order to determine the modal gain, we need the confinement factor $\Gamma$ in the waveguide. At ambient conditions for the set of two quantum wells, we take $\Gamma=$ 0.0219 (following Ref. [7]).

The confinement factor depends on the geometry of the active layer, on refractive indices and on the emission wavelength $\lambda$. The dominant effect of pressure and temperature on $\Gamma$ occurs through the changes of $\lambda$; however, there is some small effect due to the refractive index contrast varying with pressure and temperature but it was shown to be negligible in Refs. [8] and [9]. In Refs. [10] and [11], the five-layer dielectric slab waveguide model was used to determine the pressure variation of $\Gamma$. For the $1540 \mathrm{~nm}$ telecom laser, the authors obtained $\mathrm{d} \Gamma /(\Gamma \mathrm{d} p)=0.114 \mathrm{GPa}^{-1}$ and this is the value that we adopt for our laser. The corresponding temperature variation of $\Gamma$ (occurring also through the changes in $\lambda$ ) has been taken as $\mathrm{d} \Gamma /(\Gamma \mathrm{d} T)=-4.5 \times 10^{-4} \mathrm{~K}^{-1}$. Summarizing, we use the following formula for temperature and pressure dependence of the confinement factor $\Gamma$ :

$$
\begin{gathered}
\Gamma(p, T)=0.0219 \times[1+0.114 p \\
\left.-4.5 \times 10^{-4}(T-293)\right],
\end{gathered}
$$

where $p$ and $T$ are given in GPa and $\mathrm{K}$, respectively.

We have to relate the carrier concentration to the current density. In telecom lasers it is established that the 
two major contributors to threshold current are: (i) the radiative recombination and (ii) the Auger recombination

$$
I_{\mathrm{th}}(p, T)=I_{\mathrm{rad}}(p, T)+I_{\mathrm{Aug}}(p, T) .
$$

The radiative recombination is proportional to the square of the carrier concentration (we assume charge neutrality of the quantum well i.e. $n=p)$ :

$$
I_{\text {rad }}(p, T)=B(p, T) \times n^{2}(p, T),
$$

where

$$
\begin{aligned}
B & =\frac{2 \pi e^{2} n_{1} E_{p} L_{z} E_{\mathrm{g}}}{3 m_{0}^{2} c^{3} \varepsilon_{0} \varepsilon_{r} k_{\mathrm{B}} T} \frac{1}{m_{\mathrm{hh}}+m_{\mathrm{c}}} \\
& \times\left(\frac{m_{\mathrm{hh}}}{m_{\mathrm{hh}}+m_{\mathrm{c}}}+\frac{m_{\mathrm{lh}}}{m_{\mathrm{lh}}+m_{\mathrm{c}}}\right),
\end{aligned}
$$

and depends on pressure and temperature mainly through the factor $E_{\mathrm{g}} / k T$. In $980 \mathrm{~nm} \mathrm{InGaAs/GaAs}$ lasers (where the threshold current is dominated by radiative transitions) it has been shown [12] that the radiative current is approximately proportional to $k T$. Pressure measurements of $980 \mathrm{~nm}$ lasers revealed [12] a very small variation of threshold current with pressure. Therefore in the present paper we assume a linear $k T$ dependence of $I_{\text {rad }}$ and we assume that it does not change with pressure.

The Auger recombination is proportional to $n^{3}$ :

$$
I_{\text {Aug }}(p, T)=C(p, T) \times n^{3}(p, T),
$$

where the coefficient $C$ depends on the process involved $[11,13,14]$ but in general it is proportional to $\exp (-\Delta E / k T)$, where $\Delta E$ is the activation energy that increases with band gap. This is why the Auger recombination decreases rapidly with increasing pressure and decreasing temperature.

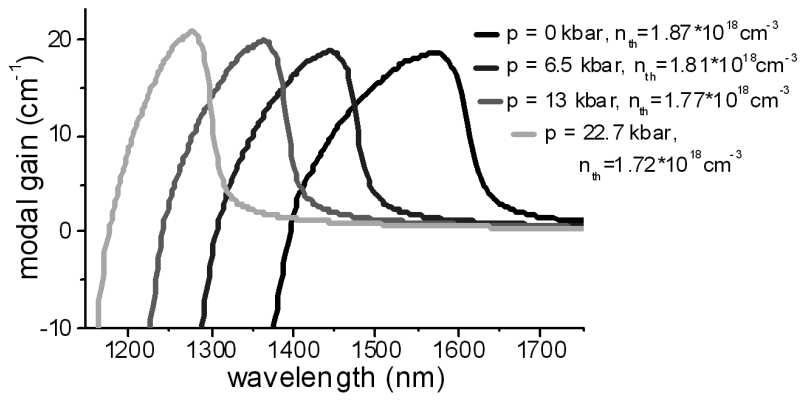

Fig. 1. Modal gain at threshold for the InGaAsP laser for different pressures (at room temperature) and corresponding threshold concentrations $n_{\text {th }}$.

At room temperature and ambient pressure about $50 \%$ of the threshold current is due to radiative recombination and $50 \%$ to the Auger recombination. The carrier concentration at threshold $n_{\mathrm{th}}(p, T)$ obtained from Eq. (7) is roughly proportional to $k T$ and slowly decreasing with pressure. Assuming typical for such lasers value of radiative threshold current ( $23 \mathrm{~mA}$ ) at $300 \mathrm{~K}$ and ambient

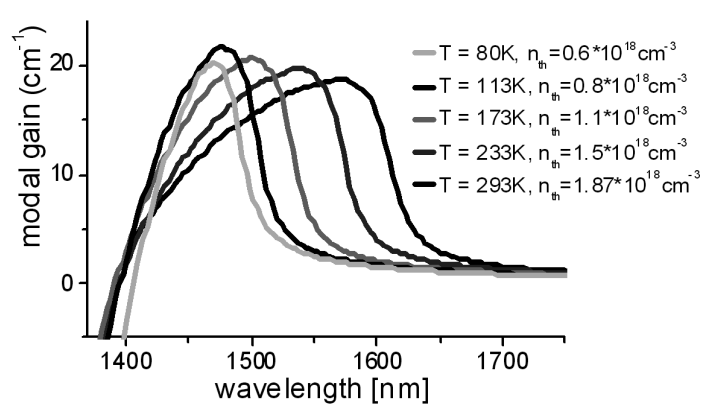

Fig. 2. Modal gain at threshold for the InGaAsP laser for different temperatures (at ambient pressure) and corresponding threshold concentrations $n_{\text {th }}$.

pressure and using the typical temperature dependence of threshold current, we are able to determine its radiative component. This, in turn, allows us to determine the carrier concentration for other pressures and temperatures. The corresponding threshold concentrations are depicted in the legend of Fig. 1 and Fig. 2.

\section{Results and discussion}

For the particular structure studied in this paper, the wavelength can be tuned by about $300 \mathrm{~nm}$ for pressures ranging from 0 to 22.7 kbar (see Fig. 3) and by $100 \mathrm{~nm}$ changing the temperature from $300 \mathrm{~K}$ to $80 \mathrm{~K}$ (see Fig. 4).

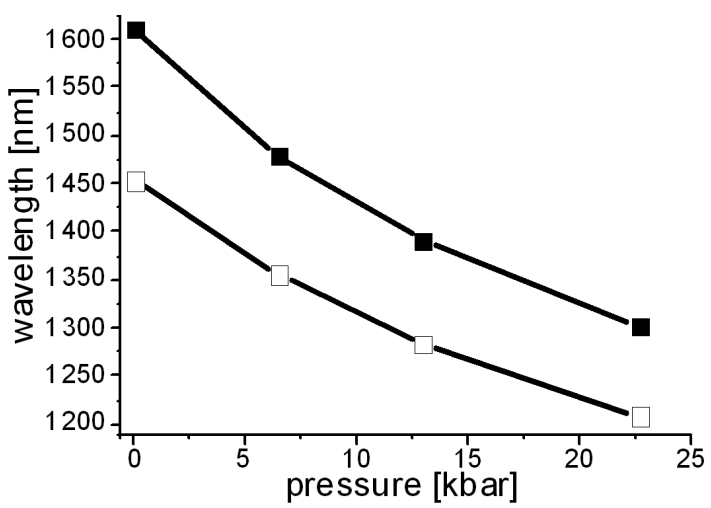

Fig. 3. Predicted range of the positive net gain vs. pressure for temperature $293 \mathrm{~K}$. This represents the estimate of the total tuning range achievable with pressure and external grating. Empty symbols denote the short-wavelength zeros of the net gain, whereas full symbols denote the long-wavelength zeros of the net gain.

The maximum gain must be equal to the sum of internal losses and mirror losses. In Figs. 1 and 2 the internal optical loss has been assumed to be $11 \mathrm{~cm}^{-1}$ [7] and we neglect its temperature and pressure variation. The internal losses (like the free-carrier absorption or intra-valence band absorption) may change significantly with the emission wavelength but as long as they are much smaller than the maximal gain, their variation does not 


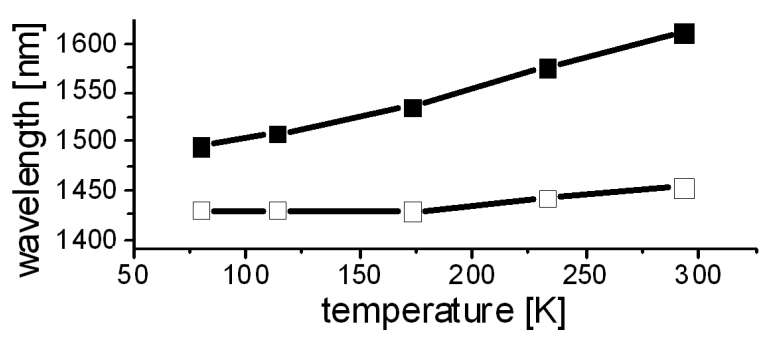

Fig. 4. Theoretical predictions for the range of the positive net gain vs. temperature at zero pressure. This represents the estimate of the total tuning range achievable with temperature and external grating. Empty and full symbols denote, respectively, the short-wavelength and the long-wavelength zeros of the net gain.

affect the width of the positive net gain. Figures 3 and 4 represent the total possible tuning range, if we combine pressure or temperature tuning with external cavity one. If we compare them, we can see that pressure tuning allows for a wider overall tuning range than temperature tuning when combined with tuning by grating. In fact, the narrowing of the gain curves at low temperatures is so significant that we lose the tuning range (compared to the room-temperature case).

\section{Summary and conclusions}

The studies of the external cavity tuning with temperature and pressure have been performed for a quantum-well laser based on InGaAsP quantum well. These studies are based on gain calculations in the framework of the $8 \times 8 k \cdot p$ model. It turns out that lowering the temperature is not effective in extending the overall tuning range due to the rapid narrowing of the threshold gain at low temperatures. This is mainly caused by the drop of threshold concentration. On the other hand, tuning at high pressure allows an increase of the overall tuning range from $160 \mathrm{~nm}$ up to $390 \mathrm{~nm}$. We have also investigated the influence of external factors on the nonradiative and radiative currents. We find out that the nonradiative current amounts to about $50 \%$ of the total current at ambient conditions and decreases to about $20 \%$ at the pressure of $2 \mathrm{GPa}$.

\section{Acknowledgments}

This work was supported by Polish Ministry of Science through grant no. N R02 000206.

\section{References}

[1] Cunyun Ye, Tunable External Cavity Diode Lasers, World Scientific, Singapore 2004.

[2] W. Trzeciakowski, A. Bercha, F. Dybala, R. Bohdan, P. Adamiec, O. Mariani, Phys. Status Solidi B 244, 179 (2007).

[3] http://www.nextnano.de .

[4] L.A. Coldren, S.W. Corzine, Diode Lasers and Photonic Integrated Circuits, Wiley, New York 1995.

[5] I. Vurgaftman, J.R. Meyer, L.R. Ram-Mohan, J. Appl. Phys. 89, 5815 (2001).

[6] P.J.S. Heim, Z.F. Fan, S.-H. Cho, Keeyol Nam, M. Dagenais, F.G. Johnson, R. Leavitt, Electron. Lett. 33, 1387 (1997).

[7] S.H. Cho, C.C. Lu, M. Hovinen, K. Nam, V. Vusirikala, J.H. Song, F.G. Johnson, D. Stone, M. Dagenais, IEEE Photon. Technol. Lett. 9, 1081 (1997).

[8] S.R. Jin, R. Fehse, S.J. Sweeney, G. Knowles, A.R. Adams, E.P. O'Reilly, H. Riechert, S. Illek, A.Yu. Egorov, P.J.A. Thijs, T. Uchida, T. Fujii, Electron. Lett. 38, 325 (2002).

[9] M. Hawley, Ph.D. Thesis, University of Surrey, 1994.

[10] B. Gonul, Semicond. Sci. Technol. 14, 648 (1999).

[11] B. Gonul, M. Oduncuoglu, Semicond. Sci. Technol. 19, 23 (2004).

[12] M. Maziarz, B. Piechal, A. Bercha, R. Bohdan, W. Trzeciakowski, J.A. Majewski, Acta Phys. Pol. A 112, 437 (2007).

[13] A. Haug, Semicond. Sci. Technol. 7, 1337 (1992).

[14] A. Haug, Electron. Lett. 26, 1415 (1990). 